Meta

Journal des traducteurs

Translators' Journal

\title{
Langue spécialisée et technolecte : quelles relations ?
}

\section{Leila Messaoudi}

Volume 55, numéro 1, mars 2010

Le parcours du sens : d'une langue à l'autre — Mélanges offerts à André Clas

The Way of Meaning: From a Language to Another - Collection of Articles Offered to André Clas

URI : https://id.erudit.org/iderudit/039607ar

DOI : https://doi.org/10.7202/039607ar

Aller au sommaire du numéro

Éditeur(s)

Les Presses de l'Université de Montréal

ISSN

0026-0452 (imprimé)

1492-1421 (numérique)

Découvrir la revue

Citer cet article

Messaoudi, L. (2010). Langue spécialisée et technolecte : quelles relations ? Meta, 55(1), 127-135. https://doi.org/10.7202/039607ar

\section{Résumé de l'article}

Le présent article traite de questions relatives aux deux termes langue spécialisée et technolecte et du type de relations qui les lient. Un rappel de la continuité existant entre la langue générale et la langue dite spécialisée est nécessaire avant d'essayer de présenter les traits caractérisant les langues spécialisées, pour aboutir à l'idée que ces dernières auraient plutôt tendance à désigner les usages savants et écrits. Or, des domaines d'activité techniques traditionnels - et parfois même modernes - dans des sociétés peu développées usent de l'oral de façon prépondérante. Il se trouve que cet aspect n'est point recouvert par la dénomination de langue spécialisée qui accorde la priorité à la langue (au sens saussurien). L'usage du terme de technolecte a l'avantage, au vu de ses formants, de pouvoir s'appliquer aussi bien à l'oral qu'à l'écrit et revêtirait ainsi un caractère plus générique que celui de langue spécialisée.
Ce document est protégé par la loi sur le droit d'auteur. L'utilisation des services d’Érudit (y compris la reproduction) est assujettie à sa politique d'utilisation que vous pouvez consulter en ligne.

https://apropos.erudit.org/fr/usagers/politique-dutilisation/ 


\title{
Langue spécialisée et technolecte: quelles relations?
}

\author{
LEILA MESSAOUDI \\ Université Ibn Tofail, Kénitra, Maroc \\ lmessaoudi@gmail.com
}

\begin{abstract}
RÉSUMÉ
Le présent article traite de questions relatives aux deux termes langue spécialisée et technolecte et du type de relations qui les lient. Un rappel de la continuité existant entre la langue générale et la langue dite spécialisée est nécessaire avant d'essayer de présenter les traits caractérisant les langues spécialisées, pour aboutir à l'idée que ces dernières auraient plutôt tendance à désigner les usages savants et écrits. Or, des domaines d'activité techniques traditionnels - et parfois même modernes - dans des sociétés peu développées usent de l'oral de façon prépondérante. II se trouve que cet aspect n'est point recouvert par la dénomination de langue spécialisée qui accorde la priorité à la langue (au sens saussurien). L'usage du terme de technolecte a l'avantage, au vu de ses formants, de pouvoir s'appliquer aussi bien à l'oral qu'à l'écrit et revêtirait ainsi un caractère plus générique que celui de langue spécialisée.
\end{abstract}

\section{ABSTRACT}

This article deals with some matters related to two terms, specialised language and technical language, and the type of relations between them. Before attempting to present the features characterising specialised languages, it is necessary to recall the continuity between general language and the language referred to as specialised to reach the idea that specialised languages were rather inclined to indicate learned and written forms. And yet, less-developed societies, in traditional - and sometimes even modern - technical domains of activity, make use of the oral language predominantly. However, this aspect is by no means covered by the term specialised language which prioritises language as a system (in the Saussurian sense). The use of the term technical language, in the view of its creators, has the advantage of being applicable to the oral as well as to the written language and could thus assume a more generic character compared to that of specialised language.

\section{MOTS-CLÉS/KEYWORDS}

langue spécialisée, technolecte, domaines, écrit/oral, développement specialised language, technolect, domains, written/oral language, development

Nous entendons souvent parler de langue technique et scientifique, par opposition à langue générale, de langue spécialisée, par opposition à langue commune, de langue de spécialité, par opposition à langue courante, ou encore de langue fonctionnelle, par opposition à langue poétique, littéraire, ordinaire, etc. Ces oppositions impliquent que l'on pose l'existence d'une distinction nette entre, en quelque sorte, deux types de langues. On peut alors se demander si, sur le plan purement linguistique, cette distinction a lieu d'être et si elle est réellement pertinente: les langues spécialisées possèdent-elles véritablement un fonctionnement propre, différent de celui de la langue générale? Constituent-elles un noyau linguistique à règles particulières? Au sein d'une même langue ou bien en mobilisant plusieurs langues comme ce peut être 
le cas en contexte plurilingue où les usagers d'une profession puisent dans les ressources de l'une ou l'autre langue (par exemple, à l'arabe marocain et au français au Maroc [Messaoudi: 2002; 2004])?

Certes, à écouter des échanges linguistiques de deux chimistes dans leur laboratoire ou de deux mécaniciens dans leur garage, un tiers profane ne comprendrait goutte à leur parlure: pour lui, ils s'entretiennent d'un domaine qui lui est inaccessible, dans une langue qu'il identifie comme étant la sienne mais qui, en l'occurrence, ne lui est pas parfaitement transparente. Il reconnaîtra que c'est son incompétence dans ledit domaine qui l'empêche de saisir les propos des deux locuteurs et il conclura que ces activités, différentes des circonstances ordinaires de la vie quotidienne, possèdent un langage propre, qui lui est totalement ou partiellement étranger. Phal signalait à ce sujet:

Un physicien, un biologiste, un agronome, un économiste... ont en commun un certain nombre de mots qui, indépendamment de la difficulté de contenu, nous déconcertent et nous font dire que les savants et les techniciens parlent une langue différente de celle que nous parlons dans les circonstances de la vie quotidienne (Phal 1968: 7).

De dépeindre de façon aussi caricaturale le sentiment qu'éprouve tout un chacun, dans des circonstances peu exceptionnelles, telles que celles de se trouver dans un garage de mécanique automobile, ou dans un laboratoire pharmaceutique, ou dans un tout autre lieu dédié à une activité technique spécialisée, n'est pas dénué d'intérêt: cela permet de dessiner les contours de la notion de «langue spécialisée» et de dégager les premiers traits définitoires.

\section{Des domaines ou champs d'activités humaines spécialisées}

Il semblerait que ce soient les différents domaines ou champs d'activités humaines qui contribuent à l'existence de langages variés: une typologie de plus en plus fine de ces activités conduirait à en saisir les différents aspects et à en détecter le fonctionnement. Cette entreprise est-elle possible? Est-elle seulement envisageable? Sans prétendre à une exhaustivité qui ne peut être qu'utopique, l'on pourrait tracer une grille dans laquelle seraient rangées les professions exercées dans une société donnée, à un moment donné de son histoire. Cette tentative de classer les professions existe déjà; par exemple, en France, la nomenclature des professions et des catégories socioprofessionnelles est publiée et mise à jour régulièrement par l'INSEE ${ }^{1}$.

Et l'on peut faire varier les situations à loisir et imaginer toutes sortes de contextes dans lesquels deux paramètres seront toujours présents: le profil du locuteur en situation et les propos scientifiques et techniques. Il est évident que dans la perspective d'une approche globale, la linguistique interne de Saussure n'est guère suffisante, à elle seule, pour dégager les diverses variétés spécialisées d’une ou de plusieurs langues, car ce sont les facteurs extralinguistiques (domaines d'activités et situation) qui permettent de repérer, en premier lieu, les langues de spécialité. Spillner signale, quant à lui, l'importance des profils des locuteurs puisqu'il s'agit de spécialistes mobilisés dans une situation de communication : pour lui, «les langues de spécialité » sont constituées "par l'ensemble des éléments linguistiques qui peuvent se manifester, dans une situation donnée, lors de la communication entre spécialistes d'une même discipline» (Spillner 1982: 19-27). 
En effet, l'on peut facilement imaginer une discussion à caractère technique, entre deux pharmaciens s'entretenant de la composition d'un médicament autour d'un verre de thé, l'été, au bord de la mer. Même si la situation à laquelle appartiennent les propos n'est pas à caractère proprement technique, le domaine auquel renvoient tacitement - et de commun accord - les deux interlocuteurs est celui de la fabrication des médicaments et leur composition. Ils devisent du domaine pharmaceutique tout en étant loin de l'officine. Ce qui démontre l'importance du domaine qui peut être déterminant pour la nature des propos échangés même si la situation est tout autre et présente peu de liens avec le domaine concerné!

D’emblée, ce ne sont point les traits linguistiques qui seront retenus dans un premier temps pour identifier un vocable, une expression ou une phrase comme revêtant un caractère technique ou spécialisé, mais bien le domaine technique d'où ils sourdent et la situation à caractère technique dans laquelle ils se trouvent proférés ou bien à laquelle ils réfèrent. Quemada (1978: 1148-1149) note à ce sujet: «l'appartenance d'un vocable à un domaine technique est acquise par le fait d'être utilisé dans une communication à thème et en situation technique. Cela suffit à lui conférer la qualité de terme technique». Mais à quoi renvoient les termes technique, scientifique et technologique qui se trouvent accolés aux langues spécialisées?

\section{Domaines scientifiques, techniques, technologiques : quelles différences?}

En dépit de multiples discours sur la science, la technique et la technologie, il n'est pas toujours aisé de définir avec précision ce que recouvrent ces branches de la connaissance, tant il est vrai que leur contenu change non seulement en fonction des époques, mais aussi en fonction des pays et des besoins, et surtout des idéologies qui les sous-tendent. Sans nous arrêter aux multiples aspects que peuvent revêtir ces appellations, notamment dans le contexte des pays du Sud, nous nous contenterons d'examiner la question dans la seule perspective linguistique qui nous intéresse ici.

Et pour commencer, l'on pourrait déplorer le flou et l'imprécision de ces dénominations. Par exemple, Phal signale l'aspect trompeur de l'appellation langue scientifique en notant:

[...] parler de «langue scientifique» sans préciser davantage, c'est ignorer:

- les différences qui existent entre le niveau de la Science fondamentale et celui de «l'application technique» (entre la géologie et la prospection minière par ex.);

- les différences qui existent, dans une même science fondamentale, entre la science mère et ses ramifications de plus en plus spécialisées (exemple: mécanique générale - mécanique des fluides - mécanique des fluides incompressibles, ou hydraulique);

- les différences qui existent entre la langue de la science qui se fait (recherche) et la langue de la science qu'on transmet (enseignement, vulgarisation) (Phal 1968: 7).

Ces ambiguïtés susciteront de nombreuses interrogations chez le linguiste, dont le souci majeur est d'être en possession de critères formels permettant de caractériser tel ou tel ensemble langagier comme étant technique ou scientifique. Or, qu'est-ce qui distingue le technique du scientifique? Faut-il les séparer de la langue générale ou commune? Possèdent-ils les mêmes traits linguistiques ou bien ont-ils chacun leurs spécificités? Certains chercheurs proposent de distinguer entre technique et 
scientifique. C'est la position de Descamps et Phal, telle qu'elle ressort des définitions suivantes:

- Le vocabulaire technique est spécifique (propre à une science ou à une technique donnée). C'est essentiellement un vocabulaire de désignation qui fournit les nomenclatures, les terminologies [...];

- Le vocabulaire scientifique n'est pas spécifique d'une science ou d'une technique donnée. Il se situe entre le vocabulaire «usuel» de la langue quotidienne et le vocabulaire technique. Il comprend des mots de sens très général qui trouvent à s'employer dans toutes les spécialités à un niveau fondamental (Descamps et Phal 1968: 12).

C'est aussi, à quelques nuances près le point de vue adopté par Guilbert qui souligne:

La considération de la personne du locuteur, et non plus, du seul référent est de nature à nuancer l'analyse. Le terme scientifique, en effet, est employé dans un milieu homogène de spécialistes de même culture, de même formation. La communication se situe à un haut niveau d'élaboration conceptuelle et de rigueur dans l'analyse. Elle fait appel de préférence à l'énonciation écrite [...]. Les techniciens, par contre, représentent une catégorie de locuteurs non homogène, pouvant englober ceux qu'on nomme les cadres et les ouvriers [...]. Les locuteurs techniciens, en effet, constituent une catégorie socioprofessionnelle moins close que celle des hommes de science [...] (Guilbert 1973: 15).

Il est à noter que si pour Descamps et Phal (1968), c'est le domaine du savoir qui permet de procéder à la distinction entre les deux, de telle façon que le scientifique - plus large et par conséquent, plus abstrait - soit en mesure d'englober le second; pour Guilbert (1973), il en va tout autrement, puisque c'est en la personne du locuteur que réside la différence: l'ensemble des locuteurs scientifiques constitue un monde clos, homogène et qui a le plus souvent recours à l'expression écrite; quant au monde des techniciens, il est plus ouvert, hétérogène et plus axé sur les aspects pratiques et concrets.

Nonobstant l'importance de ces critères pour séparer le scientifique du technique tout en les différenciant de la langue commune ou générale, les doutes du linguiste demeureront. Ils ne s'esquiveront que devant des traits formels, difficilement contestables. Ainsi, le problème reste entier, puisqu'il n'est pas aisé de tracer des frontières nettes et parfaitement étanches entre le technique et le scientifique qui ne cesseront de se chevaucher; en outre, il n'est même pas certain que l'on puisse les isoler de la langue générale, difficulté que ressentent avec acuité les confectionneurs des ouvrages lexicographiques. Quemada (1978: 1148) note à ce propos: «Il faut reconnaître qu'une délimitation rigoureuse entre technique et non technique se révèle toujours délicate en matière de langage, ce dont témoignent les faiseurs de dictionnaires depuis qu'ils opèrent cette discrimination nécessaire». Au demeurant, la distinction n'est pas toujours aisée entre le technique et le scientifique puisqu'ils s'enchevêtrent souvent même si les aspects abstraits et théoriques paraissent être du ressort du scientifique, tandis que les volets concrets et appliqués semblent plus appartenir au technique.

Par ailleurs, les termes technique et technologie sont employés soit de manière distincte, soit de manière synonymique. Le plus souvent, cependant, l'on constate que le second a tendance à supplanter le premier. D’après Daumas, 
les techniques sont les procédés et méthodes de création de biens matériels de consommation, d'équipement ou de communication [...]. Elles utilisent des matériaux qui sont toujours d'origine naturelle: leurs chaînes plus ou moins complexes d'extraction, de transformation et de mise en œuvre fournissent des produits qui possèdent, en général, des caractéristiques physiques qui les rendent directement perceptibles par l'homme mais dont certains, tels les courants électriques faibles, ne peuvent être perçus qu'indirectement par lui (Daumas 1981: 4).

Et si le terme de technologie concurrence actuellement celui de technique, il le devance notamment dans les domaines de l'informatique et des télécommunications, à travers Internet. On parle de TIC (technologie de l'information et de la communication) et on n'use plus de NTIC (N correspondait à nouvelles [technologies]). Il est intéressant de remarquer la probable et attendue disparition du sens quavait le mot technologie au moment de son apparition: au XVII siècle, il renvoyait au discours sur les techniques, «c'est-à-dire la science raisonnée des différentes techniques » (Daumas 1981: 4).

\section{Domaines techniques traditionnels et modernes: deux modes d'être au monde?}

La réflexion sur ces notions n'est pas sans lien avec celle du développement, ou comme il est convenu de le nommer actuellement, le développement durable (Messaoudi: 2007). Sans prétendre aborder de manière approfondie cette question hautement intéressante en raison de sa portée tout à la fois philosophique et sociétale, nous voudrions nous arrêter quelque peu aux notions de technique et technologie, conçues toutes deux comme ensembles de savoirs et de savoir-faire. Souriau (1976: 91) s'interrogeait à juste titre: "Quelle société humaine pourrait-elle donc [sic] survivre sans fabriquer des objets et des outils, sans mettre au point un raisonnement organisationnel pour produire, reproduire, sauvegarder et éventuellement améliorer sa manière de vivre et le cadre de cette vie?» Et réfléchissant sur l'univers maghrébin, elle ne manquait pas de souligner:

Aboutir, à partir de l'environnement maghrébin, comme cela a été fait des siècles avant l'industrialisation, à l'exploitation des ressources naturelles, à la reproduction de la force de travail, à des activités pour tous; ou de façon plus concrète, obtenir un couscous complet pour une famille élargie, un tissage à l'épreuve des intempéries, un univers domestique adéquat, une improvisation au luth, un artisanat commercialisé, un réseau trans-saharien fonctionnel, l'exploitation conjuguée du palmier et de l'olivier, l'irrigation en climat aride; et, par ailleurs encore, un partage successoral régulier, un mariage fécond, une prière licite, etc.; tout cela ne serait pas le fruit d'une société technicienne, d'une technologie? (Souriau 1976: 91)

Tout en appréciant à sa juste valeur cette conception valorisante d'une société technicienne, d'une technologie comme l'entend Souriau (1976), et que l'on désigne communément par savoirs et savoir-faire locaux, l'acception qui s'impose actuellement est celle de supports scientifiques avancés, s'appuyant sur des techniques sophistiquées et sur des technologies (TIC) de plus en plus accessibles. En témoigne le nombre de centres et de cybercafés, y compris dans des contrées fort éloignées et à caractère rural: par exemple, dans certains douars au Maroc et dans le péri-urbain des grandes villes, les jeunes, ne souhaitant pas rester en dehors de la modernité 
déferlante, trouvent moyen de se connecter à Internet, d'autant que les tarifs facturés à l'heure (ou même à la demi-heure) sont peu coûteux; pour donner un ordre de grandeur, ils sont six fois moins chers que ceux appliqués dans certains cybers en France.

$\mathrm{Au}$-delà du fait que toute société connaît, au cours de son histoire, diverses étapes de développement et que le Maroc et le Maghreb ne font pas exception, nous sommes loin de partager totalement les opinions de nostalgiques qui regrettent les sociétés rudimentaires, se suffisant de peu et prônant le principe de la pauvreté, richesse des peuples (Tevoedjre 1978). S’il est entendu que l'évolution non maîtrisée de la technologie peut avoir des répercussions regrettables sur l'être humain et son environnement, il n'en demeure pas moins que l'on ne peut s'extraire de cette évolution et l'ignorer.

Ainsi, s'il est utile pour l'environnement et pour la mémoire collective de conserver les techniques traditionnelles, il est absolument nécessaire de s'inscrire dans la modernité avec tout ce qu'elle entraîne comme évolution technique et révolution technologique, s'accompagnant, autant que faire se peut, par des bouleversements systémiques des langues. Les pays du Sud ne peuvent en faire l'économie ni en faire les frais: ils subissent, qu'ils le veuillent ou non, le poids de la mondialisation, de la globalisation. Ils doivent donc s'approprier les techniques et technologies tout en tirant profit des savoirs locaux et en les préservant, au prix de médiations linguistiques de toutes sortes.

Dès lors que l'on opte pour une continuité et non une rupture entre techniques dites traditionnelles et celles dites modernes, dès lors que l'on conçoit la coexistence des deux au sein d'un même environnement social, les ensembles langagiers spécialisés désignent, pour nous, aussi bien les savoirs locaux traditionnels que les savoirs de pointe des secteurs modernes. Cette conception nous est dictée par le terrain que nous connaissons le mieux: celui du Maghreb et, plus spécifiquement, celui du Maroc où les deux modes de vie traditionnel et moderne se côtoient de manière visible et manifeste sur le plan du costume, du transport, de l'art culinaire, du maniement des langues, etc. De fait, en raisonnant sur les langages spécialisés, on ne peut ignorer ces deux modes d'être au monde, traditionnel et moderne, qui trouvent leur expression à travers des moyens linguistiques appropriés. L'on peut s'interroger sur ces moyens linguistiques: sommes-nous face à des langues spécialisées? Cette appellation est-elle pertinente? Avant de répondre à ces questions, nous essaierons de récapituler les traits caractéristiques des langues spécialisées.

\section{Les langues spécialisées : quels traits caractéristiques?}

La langue spécialisée, comme nous l'avons vu plus haut, est utilisée pour servir à la communication entre spécialistes de tel ou tel domaine scientifique ou technique, le plus souvent en situation professionnelle:

- Une langue spécialisée ne constitue pas une langue à part: elle utilise les ressources (morphologiques, syntaxiques, lexicales) des langues communes mobilisées pour l'expression spécialisée de tel ou tel domaine de l'activité humaine. Cusin-Berche (1995: 42) soulignait à ce sujet « [qu']il n'existe pas une langue technique opposable à une langue standard, mais des usages discursifs et lexicaux propres à chaque domaine d'activité ». On peut donc la définir comme «l'usage d'une langue naturelle 
pour rendre compte techniquement de connaissances spécialisées» (Lerat 1994: 21);

- Elle ne se réduit pas au jargon ou à la terminologie (Messaoudi 1990; 2003; Lerat 1994) : elle utilise des dénominations spécialisées (les termes), y compris des symboles non linguistiques, dans des énoncés mobilisant les ressources ordinaires d'une langue donnée;

- Le degré de technicité dans la formulation est variable selon les besoins de la communication, qui va de la haute technicité à la vulgarisation, en passant par la banalisation;

- Les langues spécialisées imposent de donner la priorité à la linguistique de l'écrit, en élargissant la notion de "plurisystème graphique» (Lerat 1994: 29). L'usage d'alphabets différents (grec ou latin, par exemple) permet de distinguer des notions ou des objets. Par exemple, une antenne en $T$ (où le caractère en majuscule de l'imprimerie renvoie à la forme de l'antenne) est distincte de l'antenne parabolique. On peut noter, en passant, que la traduction de cette expression en arabe pose problème et la solution adoptée est de conserver le $\mathrm{T}$ dans sa forme latine qui renvoie à la forme sémiotique de ce type d'antenne. Ce qui donne en arabe, dans le dictionnaire: T هو ائية على شكل ;

- L'avis du spécialiste du domaine est l'ultime recours pour valider le caractère spécialisé ou non d'un énoncé, d'un terme..., comme le signale Lerat (1994: 47): «En fin de compte, le critère des critères est l'avis du spécialiste, dont on ne saurait raisonnablement tenter de faire l'économie».

Sur le plan linguistique, il n'est pas inutile de rappeler que les langues spécialisées ne constituent pas des sous-systèmes linguistiques autonomisables comme le signale Lerat (1994): une continuité manifeste avec les langues communes de référence et leurs ressources s'impose. Les langues spécialisées se présentent comme des plurisystèmes qui sont, selon Lerat, caractérisés par:

- Des signes non linguistiques au sein même de leurs énoncés (contrairement aux représentations graphiques) [...];

- Une morphologie composite, accueillante aux alternances de morphèmes, aux emprunts dénominatifs et aux formules complexes, incluant du lexical général et du lexical spécifique (comme en chimie le couple -ique / -ate) [...];

- Une syntaxe qui est tout à fait celle des langues de référence, mais avec des prédilections en matière d'énonciation (comme le fameux style impersonnel des sciences) et des phraséologies professionnelles (comme les formules stéréotypées des administrations) $[\ldots]$;

- Une linguistique de l'écrit [...];

- L'universalité potentielle des notions scientifiques et techniques (Lerat 1994: 2829).

Au vu des traits cités ci-dessus, un trait nous semble important, car il interpelle la distinction communément admise en linguistique, celle qui existe entre l'écrit et l'oral. De toute évidence, les langues spécialisées privilégient l'écrit. L'oral est souvent passé sous silence. Or, une remarque importante s'impose: les sociétés à tradition orale s'approprient les techniques, font l'apprentissage de métiers traditionnels et modernes, sans avoir recours à l'écrit. Les jeunes apprentis en mécanique automobile (et même des mécaniciens fort performants), au Maroc, sont le plus souvent analphabètes mais apprennent le métier, à travers un langage oral, en même temps que des procédures et des gestes techniques, sans jamais avoir été à l'école! L'on s'aperçoit que l'appellation langue spécialisée davantage employée pour la langue savante écrite 
ne peut guère être utilisée en ce contexte. C'est la raison pour laquelle, notre préférence sur le plan terminologique va à technolecte qui désigne tout ensemble langagier spécialisé, qu’il soit écrit ou oral.

\section{Le terme de technolecte: un caractère générique?}

Claude Hagège (1982) a été l'un des premiers à utiliser ce terme. Nous avons déjà eu l'occasion de donner quelques détails à ce sujet (Messaoudi $1990 ; 1995 ; 2000 ; 2002$ ). Le technolecte est conçu comme un ensemble d'usages lexicaux et discursifs, propres à une sphère de l'activité humaine. Ainsi, les productions écrites et orales, englobant la terminologie savante, les textes de haute scientificité, mais aussi le vocabulaire banalisé et la terminologie populaire viendront se ranger dans le technolecte. Il ne s'agit pas d'une langue à part, opposée à la langue ordinaire, comme le supposerait l'emploi de langue de spécialité.

Rappelons que le technolecte n'est pas synonyme de terminologie ou de jargon. Bien qu'il contienne le niveau lexical, il ne peut y être réduit. Il englobe aussi des usages discursifs. À la différence de la terminologie, le technolecte n'est pas essentiellement paradigmatique. Toutefois, nous n'omettrons pas de mentionner que Lerat (1994: 18) a émis quelques réserves au sujet de cette appellation en notant: «Le français de l'automobile n'est en aucune façon une sorte de langue ou de dialecte, et l'usage de termes analogiques comme "technolecte" induit un parallélisme fallacieux".

À notre sens, l'emploi de ce terme se justifie par la transparence sémantique des éléments qui le composent. Rappelons que selon le Dictionnaire de linguistique et des sciences du langage de Dubois, Giacomo et al. (1994: 275) : "Dans une langue, le lecte désigne l'ensemble de traits linguistiques différenciés qu'on peut regrouper en une structure et qu'on réfère à une couche sociale, à un groupe professionnel»; il s'agit bien d'usages linguistiques, aussi bien écrits qu'oraux spécifiques, propres à un groupe professionnel, agissant dans un domaine spécialisé.

L'élément techno- réfère à un domaine de spécialité, en priorité technique, mais pas forcément. Rien ne nous interdirait de parler du technolecte de la critique littéraire, par exemple. Il faut reconnaître, toutefois, que ce terme a plutôt désigné les usages linguistiques spécifiques à un domaine technique ou scientifique. Jusqu'à présent, l'intérêt a surtout porté sur la langue fonctionnelle en milieu professionnel (Cusin-Berche 1995).

Le terme technolecte vient prendre place logiquement dans le paradigme dialecte, interlecte, idiolecte, sociolecte, etc. sans pour autant désigner une langue à part. Est-il utile de rappeler qu'un dialecte n'est pas autonome? Il est toujours rattaché à une langue, à un système dont il est dépendant. Des deux appellations, celle de langue spécialisée, confinée à la langue écrite à caractère savant, celle de technolecte est la plus neutre: elle ne réfère a priori ni à l'écrit ni à l'oral, ni à la langue ni au dialecte, ni à un niveau normalisé de la terminologie, mais désigne de manière objectivante tout ensemble langagier appartenant à un domaine spécialisé. La définition que nous pourrions en donner est la suivante: c'est un savoir-dire, écrit ou oral, verbalisant, par tout procédé linguistique adéquat, un savoir, ou un savoir-faire, dans un domaine spécialisé.

Pour conclure, nous pourrions insister sur le fait que le terme de technolecte revêt un caractère générique et présente l'avantage d'englober tous les usages linguistiques 
des domaines spécialisés, qu'ils soient normalisés ou non, écrits ou oraux, savants ou populaires, traditionnels ou modernes, techniques, scientifiques ou technologiques.

\section{NOTE}

1. INSEE: Institut national de la statistique et des études économiques.

\section{RÉFÉRENCES}

Cusin-Berche, Fabienne (1995): De la langue ordinaire au(x) technolecte(s). In: Jacques Anis et Fabienne Cusin-Berche, dir. Difficultés linguistiques des jeunes en formation professionnelle courte. Diagnostic et propositions de remédiation (Actes du Colloque international de Paris X - Nanterre, 19-21 décembre 1994). Nanterre: Linx , 40-50.

Daumas, Maurice (1981): Les grandes étapes du progrès technique. Coll. Que sais-je? Paris: PUF.

Descamps, Jean Louis et Phal, André (1968): La recherche linguistique au service de l'enseignement des langues de spécialité. Le français dans le monde. 61:5-15.

Dubois, Jean, Giacomo, Mathée, Guespin, Louis, et al. (1994): Dictionnaire de linguistique et des sciences du langage. Paris: Larousse.

HAGÈGE, Claude (1982): Voies et destins de l'action humaine sur les langues. In: Claude HAGÈGE et Istvan Fodor, dir. La réforme des langues, histoire et avenir. Hambourg: Buske, 11-67.

Guilbert, Louis (1973): La spécificité du terme scientifique et technique. Langue française. 17:5-18.

Lerat, Pierre (1994): Les langues spécialisées. Paris: PUF.

Messaoudi, Leila (1990): Des technolectes. Approche linguistique. Application à l'arabe standard. Thèse de doctorat d'État. Paris: Paris V-Lille.

Messaoudi, Leila (1995): Traduction et linguistique. Le cas des technolectes. Traduction et interprétation des textes. Rabat: Publications de la Faculté des lettres et des sciences humaines, 5-15.

Messaoudi, Leila (2000): Opacité et transparence dans les technolectes bilingues (français arabe). Meta. 45(3):424-436.

Messaoudi, Leila (2002): Le technolecte et les ressources linguistiques. L'exemple du Code de la route au Maroc. Langage et société. 99:53-75.

Messaoudi, Leila (2003) : Études sociolinguistiques. Rabat: Okad.

Messaoudi, Leila (2004) : Les technolectes au Maroc. Fonctionnement et tendances d'évolution. Trames de langues: Usages et métissages linguistiques dans l'histoire du Maghreb. Paris: Maisonneuve Larose, 455-468.

Messaoudi, Leila (2007): Le technolecte agricole et les enjeux pour le développement. In: Najib Akesbi, Driss Benatya, Larbi Zagdouni et Ahmed Zouggari, dir. Devenir de la société rurale, développement économique et mobilisation sociale. Hommage à Paul Pascon. Rabat: Institut agronomique et vétérinaire Hassan II, Impressions El Maarif Al Jadida, 107-117.

Phal, André (1968): De la langue quotidienne à la langue des sciences et des techniques. Le français dans le monde. 61:7-11.

Quemada, Bernard (1978): Technique et langage. In: Bertrand Gille, dir. Histoire des techniques. Collection Encyclopédie de la Pléiade. Paris: Gallimard, 1146-1240.

Souriau, Christiane (1976): Choix de langues et transfert de technologie. In: Michel HuberT et Maurice Flory, dir. Annuaire de l'Afrique du Nord. Paris: CNRS-CRESM, 91-110.

SPILlNER, Bernard (1982): Pour une analyse syntaxique et stylistique des langues de spécialité du français. Les langues modernes. 76:19-27

Tevoedjre, Albert (1978): La pauvreté, richesse des peuples. Paris: Éditions ouvrières. 